\title{
A comparison of nonspecific solvent scales. Degree of agreement of microscopic polarity values obtained by different measurement methods
}

\author{
Pedro M. Mancini, ${ }^{a *}$ Claudia G. Adam, ${ }^{a, b}$ Graciela G. Fortunato, ${ }^{a}$ and Leonor R. Vottero ${ }^{a}$ \\ a Departamento de Química, Química Orgánica, Facultad de Ingeniería Química, Universidad \\ Nacional del Litoral, Santiago del Estero 2829 (3000) Santa Fe, Argentina \\ ${ }^{b}$ CONICET \\ E-mail:pmancini@fiqus.unl.edu.ar
}

\begin{abstract}
The active role of solvents in physicochemical processes in solution has long been recognized. Different solvatochromic empirical scales are aimed at describing and quantifying the nonspecific interactions at a molecular level. This work presents a new insight into the comparison of the famous $E_{\mathrm{T}}(30)$ (Dimroth-Reichardt) and $\pi^{*}$ (Kamlet, Abboud, Taft) solvatochromic scales. These parameters were tested against the data derived from theoretical solvent-induced shifts in the UV-vis spectra of the corresponding reference solutes (Matyushov et al.). In each case we centered the attention on the analysis of the degree of agreement between paired values quantified through both scales by applying the methodology described by J. M. Bland and D. G. Altman. In addition, the linear correlations are assessed.

The study reflects that (a) the scales involved in this comparison are clearly dependent on the type of probe used to quantify the solvent property, and (b) the experimental parameters, in general, do not agree with the theoretical ones. These results were related with the contributions of induction, dispersion and dipole-dipole forces to the overall solvent effect. It is expected that the results will contribute to the evaluation of the microscopic chemical scales ability to describe the solute-solvent interactions.
\end{abstract}

Keywords: Microscopic solvent properties, empirical polarity scales, degree of agreement

\section{Introduction}

The active role of solvents in physicochemical processes in solution has long been recognized. Chemists usually attempt to understand the solvent effects on chemical processes in terms of the solvent 'polarity' which is defined as the overall solvation power. A multitude of empirical single- and multi-parameter solvent scales designed on the basis of solvent-dependent 
phenomena (spectroscopic, kinetic, equilibrium) are aimed at describing and quantifying the solvation interactions at molecular level. ${ }^{1}$ Of these scales the easiest to determine are those based on the solvatochromic method. Between them, the scales based on the single parameter approaches include, inter alia, Dimroth-Richardt's $E_{\mathrm{T}}(30),{ }^{2 \mathrm{a}}$ Brooker's $\mathscr{\mathscr { R }}_{\mathrm{R}}{ }^{2 \mathrm{~b}}$ Walter's $\varepsilon_{\mathrm{K}},{ }^{2 \mathrm{c}}$ Brownstein's $S,{ }^{2 \mathrm{~d}}$ Kosower's $Z,{ }^{2 \mathrm{e}}$ Dubois-Bienvenue's $\Phi,{ }^{2 \mathrm{f}}$ Allerhand and Schleyer's $G,{ }^{2 \mathrm{~g}}$ Knauer and Napier's $\mathbb{N}_{\mathrm{N}}{ }^{2 \mathrm{~h}}$ Dong and Winnik's $P \mathrm{y},{ }^{2 \mathrm{i}}$ Gutmann's $A N$ and $D N,{ }^{2 \mathrm{j}}$ Kamlet, Abboud and Taft's $\pi^{*},{ }^{2 \mathrm{k}}$ Buncel and Rajagopal's $\pi^{*}{ }_{\text {azo }}{ }^{21}$ and Drago's unified polarity scale $S^{\prime 2 \mathrm{~m}}$. In addition to the single parameters, some multiparametric correlation equations (either by the combination of two or more existing scales or by postulating specific parameters to account for distinct types of effects ) have been proposed to unravel the properties of the medium. Among them, extensively used approaches were described by $\operatorname{Koppel}$ and $\operatorname{Palm}(Y, P, B, E \text { parameters })^{3 \mathrm{a}}$ Krygowski and Fawcet $\left(E_{\mathrm{T}}(30), D N\right.$ parameters) ${ }^{3 \mathrm{~b}}$ Dougherty (IP, EA parameters), ${ }^{3 \mathrm{c}}$ Katritzky et al. $\left(E_{\mathrm{T}}, \varepsilon\right.$ parameters $){ }^{3 \mathrm{~d}}$ Swain et al. $(A, B \text { parameters })^{3 \mathrm{e}}$ and Kamlet et al. $\left(\pi^{*}, \alpha, \beta\right.$ parameters $){ }^{3 \mathrm{f}}$ Additionally, Abraham devised scales of solute hydrogen-bond acidity and solute hydrogen-bond basicity proposing a general solvation equation. ${ }^{4}$ Later, Catalán et al. defined the SPP, SB and SA solvatochromic scales. ${ }^{5}$

On the other hand, quantitative structure-property relationships (QSPR) models have been employed for the treatment of the solvent scales. ${ }^{6 a}$ The solvent descriptors are derived from the molecular structure, and the CODESSA program $^{6 \mathrm{~b}}$ has been applied. Furthermore, a classification and grouping of solvents and solvent scales has been proposed employing a principal component analysis (PCA). ${ }^{7}$

An exhaustive review concerning the quantification of solvent polarity has been recently reported ${ }^{8(a)}$, which includes a detailed list of solvent scales, interrelations between parameters and statistical approaches. Moreover, both a classification of solvents and a clustering of solvent scales have been performed on the basis of QSPR approach and PCA treatment. ${ }^{8 \mathrm{~b}}$ In particular, a previous contribution ${ }^{9}$ reported a comparative analysis in order to determine whether the more relevant solvent polarity/polarizability scales $\left[E_{\mathrm{T}}{ }^{\mathrm{N}}(30)\right.$ (Dimroth-Reichardt), $\pi^{*}$ (Kamlet, Abboud and Taft, KAT), Py (Dong and Winnik), $S^{\prime}$ (Drago) and SPP (Catalán et al.)] are pure descriptors of nonspecific solvent effects or if they are contaminated with specific effects. These scales were tested against the data derived from the theoretical thermodynamic analysis of solvent-induced shifts in the UV-Vis spectra of chromophores presented by Matyushov et al. ${ }^{10}$ This approach (which may be called the physical approach in contrast to the chemical one) analyzes the spectral shifts of 4-nitroanisole and 2,6-diphenyl-4-(2,4,6-triphenyl-1-pyridinio)phenolate (betaine-30) dye (which are utilized in the $\pi^{*}$ and $E_{\mathrm{T}}(30)$ scales respectively) on the basis of ideas derived from liquid state theories. The comparative analysis ${ }^{9}$ was carried out by analyzing the linear correlation between two scales (in each case the theoretical energy of the first $\pi, \pi^{*}$ electron transition of 4-nitroanisole was taken as reference) evaluating the correlation coefficient $(r)$ and the standard deviation $(S D)$. The reported results indicate that a) the $E_{\mathrm{T}}{ }^{\mathrm{N}}(30), P \mathrm{y}$ and $S^{\prime}$ parameters should be contaminated with specific hydrogen bond donor (HBD) interactions, $b$ ) 
the $\pi^{*}$ and $S^{\prime}$ scales reflect contamination with charge-transfer (CT) effects, and c) the SPP scale would be an appropriate solvent polarity parameter.

As it is well known, the application of linear regression analysis in order to compare paired measurements obtained from different methods reveals the strength of the relation between them but says nothing about the magnitude of the differences between the compared measurements. In this sense, when different measure methods exist for a single phenomenon, it is interesting to study to what extent the results obtained with these methods are equivalent. Recently, we have reported two comparison analyses referred to multiparametric empirical solvent scales for some binary solvent mixtures. ${ }^{11}$ In this direction, we have applied the methodology described by J. M. Bland and D. G. Altman ${ }^{12}$ in order to assess the agreement between two measurement techniques.

At this point our objective is to present a new insight into the comparison of the previously cited microscopic solvent-property scales. We particularly focus on the comparison of the famous $E_{\mathrm{T}}(30)$ and $\pi^{*}$ scales.

The aim of this work is to determine the degree of agreement between molecularmicroscopic polarity paired values quantified through both cited solvent scales. The results are connected with the theoretical data of solvent-induced shifts in the UV-vis spectra of the corresponding reference solutes ${ }^{10}$ related to the contributions of induction, dispersion and dipole-dipole solvent forces to the overall solvent effect. Additionally, the linear correlations are assessed. It is expected that the results will contribute to the evaluation of the microscopic chemical scales ability to describe the nonspecific solute-solvent interactions.

\section{Results and Discussion}

Matyushov et al. reported the experimental and theoretical absorption frequencies of 4nitroanisole and betaine-30 dyes in a wide number of solvents with different chemical structures. The solvent shifts were dissected into their contributions due to induction forces, permanent dipole solvation, and dispersion interactions. ${ }^{10}$ Table 1 presents (a) the normalized theoretical $E_{\mathrm{T}}{ }^{\mathrm{N}}$ theor and experimental $E_{\mathrm{T}}{ }^{\mathrm{N}} \exp$ parameters calculated from the reported $E_{\mathrm{T}}(30)$ Matyushov's data and expression $E_{\mathrm{T}}{ }^{\mathrm{N}}=\left[E_{\mathrm{T}}(30)-30.7\right] / 32.4,{ }^{1}$ and (b) the theoretical $\pi^{*}$ theor and experimental $\pi^{*}{ }_{\exp }$ values calculated from the theoretical and experimental reported shifts of 4-nitroanisole ${ }^{9,10}$ and expression $\pi^{*}=0.427[34.2-\tilde{v}]$ ( $\tilde{v}$ is the wavenumber of the UV-Vis absorption maxima) proposed by Marcus. ${ }^{13}$ Keeping in mind that the 4-nitroanisole is only one of the solutes that make up the set of selected probes used in the quantification of the $\pi^{*}$ scale, ${ }^{2(k)}$ the published $\pi^{*}$ values are additionally presented. Besides, it is worth noticing that the $E_{\mathrm{T}}{ }^{\mathrm{N}}$ exp values are in agreement with the published ones. 
Table 1. Theoretical, experimental and published $E_{\mathrm{T}}{ }^{\mathrm{N}}$ and $\pi^{*}$ solvent parameters

\begin{tabular}{|c|c|c|c|c|c|}
\hline Solvent & $E_{\mathrm{T}}^{\mathrm{N}}$ theor & $E_{\mathrm{T}}^{\mathrm{N}}($ pub)/exp & $\pi_{\text {theor }}^{*}$ & $\pi^{*} \exp$ & $\pi^{*}$ \\
\hline 1) $n$-pentane & 0,099 & 0,009 & -0.251 & $-0,106$ & -0.08 \\
\hline 2) $n$-hexane & 0,082 & 0,009 & $-0,309$ & $-0,067$ & -0.08 \\
\hline 3) n-heptane & 0,069 & 0,012 & $-0,273$ & $-0,055$ & -0.02 \\
\hline 4) $n$-octane & 0,049 & 0,012 & $-0,229$ & $-0,0166$ & 0.01 \\
\hline 5) n-decane & 0,023 & 0,009 & $-0,321$ & 0,0133 & 0.03 \\
\hline 6) $n$-dodecane & $-0,004$ & 0,012 & $-0,121$ & 0,025 & 0.05 \\
\hline 7) ciclohexane & 0,098 & 0,006 & $-0,217$ & 0.0001 & 0.00 \\
\hline 8) benzene & 0,079 & 0,111 & -0.181 & 0,560 & 0.59 \\
\hline 9) toluene & 0,057 & 0,099 & $-0,132$ & 0,503 & 0.54 \\
\hline 10) $p$-xylene & 0,186 & 0,074 & $-0,115$ & 0,466 & 0.43 \\
\hline 11) fluorbenzene & 0,182 & 0,194 & 0,120 & 0,602 & 0.62 \\
\hline 12) chlorobenzene & 0,179 & 0,188 & 0,207 & 0,696 & 0.71 \\
\hline 13) bromobenzene & 0,171 & 0,182 & 0,252 & 0,785 & 0.79 \\
\hline 14) iodobenzene & 0,386 & 0,170 & 0,278 & 0,863 & 0.81 \\
\hline 15) nitrobenzene & 0,356 & 0,324 & 0,796 & 0,884 & 1.01 \\
\hline 16) benzonitrile & 0,302 & 0,333 & 0,815 & 0,896 & 0.90 \\
\hline 17) pyridine & 0,115 & 0,302 & 0,487 & 0,888 & 0.87 \\
\hline 18) $\mathrm{CCl}_{4}$ & 0,173 & 0,052 & $-0,259$ & 0,213 & 0.28 \\
\hline 19) $\mathrm{CHCl}_{3}$ & 0,285 & 0,259 & $-0,070$ & 0,705 & 0.58 \\
\hline 20) $\mathrm{CH}_{2} \mathrm{Cl}_{2}$ & 0,287 & 0,309 & 0,181 & 0,751 & 0.82 \\
\hline 21) 1,1-DCE & 0,263 & 0,269 & 0,443 & & 0.48 \\
\hline 22) 1,2-DCE & 0,060 & 0,327 & 0,279 & 0,746 & 0.81 \\
\hline 23) 1,1,2,2-TCE & 0,383 & 0,269 & 0,176 & 0,896 & 0.95 \\
\hline 24) 2-propanone & 0,353 & 0,355 & 0,420 & 0,632 & 0.71 \\
\hline 25) 2-butanone & 0,296 & 0,327 & 0,349 & 0,615 & 0.67 \\
\hline 26) 2-pentanone & 0,255 & 0,321 & 0,563 & & 0.65 \\
\hline 27) cyclohexanone & 0,237 & 0,281 & 0,534 & 0,700 & 0.76 \\
\hline 28) $\mathrm{HCOOEt}$ & 0,231 & 0,315 & 0,357 & & 0.61 \\
\hline 29) $\mathrm{MeOAc}$ & 0,195 & 0,287 & 0,151 & 0,500 & 0.60 \\
\hline 30) EtOAc & 0,160 & 0,228 & 0,088 & 0,466 & 0.55 \\
\hline 31) $\mathrm{BuOAc}$ & 0,507 & 0,241 & 0,246 & & 0.46 \\
\hline 32) acetonitrile & 0,432 & 0,460 & 0,396 & 0,687 & 0.75 \\
\hline 33) propionitrile & 0,556 & 0,401 & 0,439 & 0,696 & 0.71 \\
\hline 34) nitromethane & 0,540 & 0,481 & 0,564 & 0,769 & 0.85 \\
\hline 35) nitroethane & 0,080 & 0,398 & 0,554 & 0,790 & 0.82 \\
\hline 36) $\mathrm{Et}_{3} \mathrm{~N}$ & 0,042 & 0,043 & $-0,170$ & 0,089 & 0.14 \\
\hline 37) diethylether & 0,153 & 0,117 & $-0,173$ & 0,248 & 0.27 \\
\hline
\end{tabular}




\begin{tabular}{lccccc}
\hline 38) THF & 0,215 & 0,207 & 0,230 & 0,560 & 0.58 \\
39) HMPA & 0,306 & 0,315 & 0,482 & 0,893 & 0.87 \\
40) DMF & 0,458 & 0,404 & 0,664 & 0,905 & 0.88 \\
41) DMA & 0,340 & 0,401 & 0,705 & 0,866 & 0.88 \\
42) NMP & 0,387 & 0,355 & 0,748 & 0,939 & 0.92 \\
43) PC & 0,490 & 0,491 & 0,706 & 0,857 & 0.83 \\
44) DMSO & 0,481 & 0,444 & 0,735 & 1,02 & 1 \\
45) methanol & 0,398 & 0,762 & 0.263 & & 0.60 \\
46) ethanol & 0,302 & 0,654 & 0.213 & & 0.54 \\
47) 1-propanol & 0,237 & 0,617 & 0.182 & & 0.52 \\
48) 1-butanol & 0,193 & 0,602 & 0.155 & & 0.47 \\
49) 1-pentanol & 0,155 & 0,568 & 0.140 & & 0.44 \\
50) 1-hexanol & 0,125 & 0,559 & 0.128 & & 0.41 \\
51) i-propanol & 0,243 & 0,546 & 0.134 & & 0.48 \\
52) i-butanol & 0,202 & 0,552 & 0.121 & & 0.40 \\
53) 2-butanol & 0,192 & 0,506 & 0.146 & & 0.40 \\
54) tert-butanol & 0,208 & 0,389 & 0.058 & & 0.41 \\
55) $\mathrm{H}_{2} \mathrm{O}$ & 0,515 & 1,00 & 0.863 & & 1.09 \\
\hline
\end{tabular}

In order to assess between-scales differences, first we evaluated the degree of agreement between Reichardt and KAT's parameters by comparing both the experimental and the theoretical values. On the other hand, each one of the solvent scales derived from chemical approaches was evaluated against the data derived from the physical approach. Moreover, complementary information was obtained removing those solvents that exhibit the biggest differences between paired measurements. All these comparisons were performed by applying the Bland-Altman (B-A) method, which focuses on the magnitude of the differences between paired measurements exclusively. The general feature of the B-A method has been well described. ${ }^{12}$ The B-A results are presented in Table 2: the bias is the average of the differences between the values quantified through both scales (average discrepancy between methods), and the agreement limits (AL) are computed from equation $\mathrm{AL}=\operatorname{bias} \pm 1.96 \times S D$ (in which $S D$ is the standard deviation of the bias). 
Table 2. Bland-Altman results. Bias $(S D)$ : average of the differences between paired comparable values and standard deviation of the bias. Limits of agreement: Bias $\pm 1.96 \times S D$

\begin{tabular}{|c|c|c|c|}
\hline \multirow{2}{*}{$\begin{array}{l}\text { Number of } \\
\text { solvents }\end{array}$} & \multicolumn{2}{|c|}{ Difference vs average } & \multirow{2}{*}{$\begin{array}{c}\text { Linear correlation } \\
r(S D)\end{array}$} \\
\hline & $\operatorname{Bias}(S D)$ & Limits of agreement & \\
\hline \multicolumn{4}{|c|}{$\pi^{*} v S E_{\mathrm{T}}^{\mathrm{N}}$} \\
\hline $55^{\mathrm{a}}$ & $0.258(0.260)$ & $-0.251 / 0.768$ & $0.537(0.257)$ \\
\hline \multicolumn{4}{|c|}{$\pi^{*}$ theor $v S E_{\mathrm{T}}^{\mathrm{N}}$ theor } \\
\hline $55^{\mathrm{a}}$ & $-0.015(0.246)$ & $-0.497 / 0.467$ & $0.716(0.231)$ \\
\hline \multicolumn{4}{|c|}{$E_{\mathrm{T}}{ }^{\mathrm{N}} v s E_{\mathrm{T}}{ }^{\mathrm{N}}$ theor } \\
\hline $55^{\mathrm{a}}$ & $0.069(0.178)$ & $-0.280 / 0.418$ & $0.562(0.178)$ \\
\hline $44^{\mathrm{b}}$ & $-0.044(0.104)$ & $-0.208 / 0.199$ & $0.766(0.096)$ \\
\hline $38^{\mathrm{c}}$ & $-0.0086(0.055)$ & $-0.117 / 0.099$ & $0.933(0.056)$ \\
\hline \multicolumn{4}{|c|}{$\pi^{*}$ vs $\pi_{\exp }^{*}$} \\
\hline $40^{\mathrm{d}}$ & $0.023(0.047)$ & $-0.068 / 0.115$ & $0.990(0.047)$ \\
\hline $38^{\mathrm{e}}$ & $0.0245(0.038)$ & $-0.049 / 0.098$ & $0.994(0.037)$ \\
\hline \multicolumn{4}{|c|}{$\pi^{*}$ vs $\pi^{*}$ theor } \\
\hline $55^{\mathrm{a}}$ & $0.342(0.167)$ & $0.015 / 0.669$ & $0.863(0.154)$ \\
\hline $44^{\mathrm{b}}$ & $0.352(0.185)$ & $-0.015 / 0.714$ & $0.853(0.171)$ \\
\hline
\end{tabular}

${ }^{\mathrm{a}}$ All solvents. ${ }^{\mathrm{b}}$ Without the protic solvents. ${ }^{\mathrm{c}}$ Without the protic solvents and excluding (35), (22), (17), (11), (31), (33). ${ }^{\mathrm{d}}$ The available data in Table $1 .{ }^{\mathrm{e}}$ The available data in Table 1 excluding (15) and (16).

The bias value must be interpreted considering whether the discrepancy is large enough to be important from the point of view of the compared dipolarity/polarizability parameters. In this sense the criterion we have adopted to consider that acceptable agreement exists between the compared scales is that bias $\leq \pm 0.10$. In all cases, the linear correlation data are additionally presented. The B-A plots are shown in Figure 1. 

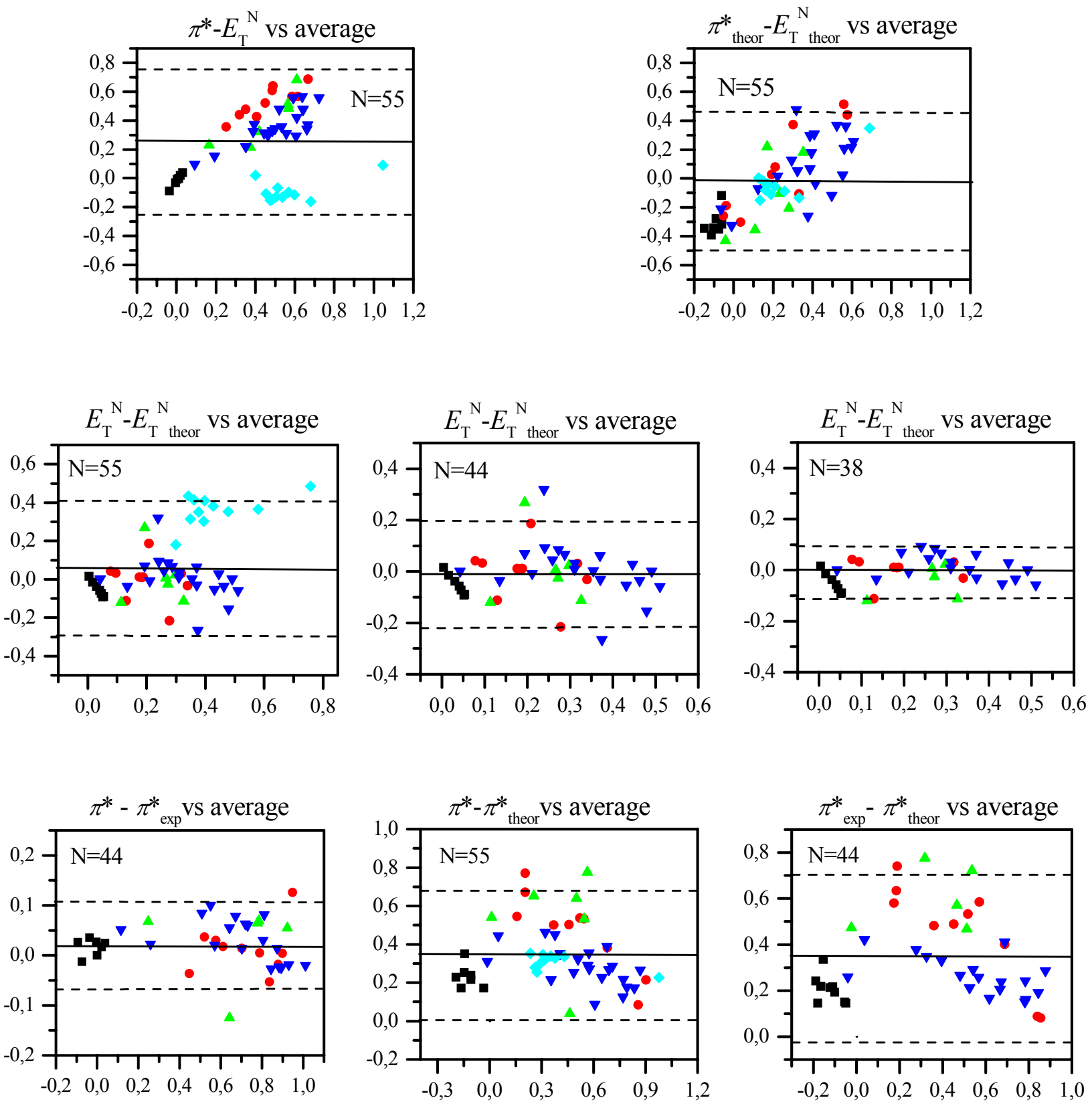

Figure 1. Bland-Altman plots. Differences between parameters against average values (solid line: bias; dashed lines: limits of agreement). Nonpolar (1-7) $\mathbf{m}$, aromatic (8-17) $\bullet$, chlorinated $(18-23) \Delta$, polar nonprotic $(24-44) \nabla$, and protic $(45-55) \diamond$ solvents.

In order to perform the scales-comparison analysis, we have investigated the degree of agreement between the commonly used published parameters as well as between the theoretical parameters. 


\subsection{Agreement between $\pi^{*}$ and $E_{\mathrm{T}}{ }^{\mathrm{N}}$}

The results (Table 2 and Figure 1) including all the explored solvents ( $N=55)$ show that (a) the bias $(0.258)$ is high $(>|0.10|)$ and are also very high, the degree of agreement between both parameters being, therefore, not acceptable; and (b) the $\left(\pi^{*}-E_{\mathrm{T}}{ }^{\mathrm{N}}\right)$ differences between paired parameters are, in most cases, higher than zero revealing that $\pi^{*}$ values tend to be higher than $E_{\mathrm{T}}{ }^{\mathrm{N}}$ values. Very high positive differences $(>0.55)$ between parameters are shown by aromatic [nitrobenzene (15), iodobenzene (14), bromobenzene (13), pyridine (17), benzonitrile (16)], polychlorinated [1,1,2,2-TClE (23)] and the polar non-protic [NMP (42), DMSO (44), HMPA (39)] solvents. Moderate negative differences are observed for the protic solvents methanol (45) and isopropanol (52). The differences are close to zero for non-polar solvents. On the other hand, the correlation coefficient $(r=0.537)$ shows that the $\pi^{*}$ and $E_{\mathrm{T}}{ }^{\mathrm{N}}$ parameters are not linearly related.

Note that the $\pi^{*}$ values tend to be higher than those of $E_{\mathrm{T}}{ }^{\mathrm{N}}$ except for some polar nonprotic solvents and most of the protic ones, leading to the high positive value of the bias. As it is well known, the solvent polarizability is better recognized by KAT than by the D-R parameter whereas, at the same time, the hydrogen-bond acceptor (HBA) character of the betaine-30 generates increased values of the corresponding parameter. $1,9,14,15$

In agreement with what was reported in other studies, ${ }^{1,14,5(a)}$ results show that the major positive differences between the published parameters are revealed by aromatic, polychlorinated and some polar nonprotic solvents, whereas the most important negative differences are revealed by protic solvents.

At this point it is of interest to corroborate which are the experimental behavior patterns that originate this disagreement. In this direction and taking into account the theoretical thermodynamic analysis presented by Matyushov et al. ${ }^{10}$ the degree of agreement between $\pi^{*}$ theor and $E_{\mathrm{T}}{ }^{\mathrm{N}}$ theor parameters was explored.

\subsection{Agreement between $\pi^{*}{ }_{\text {theor }}$ and $E_{\mathrm{T}}{ }^{\mathrm{N}}$ theor}

The results presented in Table $2(\mathrm{~N}=55)$ reveal that, although the mean difference is close to zero (bias=-0.015), the degree of agreement is not acceptable because the limits of agreement are very high $(\mathrm{AL}=-0.497 / 0.467)$. In Figure 1, the B-A plot clearly shows the great variation of the differences although the low average discrepancy between the scales. The linear relationship among the data is poor $(r=0.716)$.

It can be observed that, in general, a) for the protic solvents (with the exception of water) the differences are close to zero $\left(\pi^{*}\right.$ theor $\approx E_{\mathrm{T}}{ }^{\mathrm{N}}$ theor $)$; b) for nonpolar solvents the differences are negative; and c) for the rest of the solvents the differences hold a wide range of values. It is worth mentioning that these results reflect differences with respect to the comparison between the published parameters. 
On the one hand, the major positive differences $\left(\pi^{*}\right.$ theor $>>E_{\mathrm{T}}{ }^{\mathrm{N}}$ theor $)$ are exhibited by aromatic solvents [benzonitrile (16), nitrobenzene (15), pyridine (17)], by highly polar non-protic solvents [nitroethane (35), NMP (42), DMA (41)], and also by the protic solvent $\mathrm{H}_{2} \mathrm{O}$ (it can be pointed out that these differences are lower than those observed between the published values). On the other hand, the major negative differences $\left(\pi^{*}\right.$ theor $<<E_{\mathrm{T}}{ }^{\mathrm{N}}$ theor $)$ are exhibited by the halogenated solvents $\mathrm{CCl}_{4}$ (18) and $\mathrm{CHCl}_{3}$ (19) and also by the $n$-hexane (2), $n$-pentane (1), $n$-decane (5), and n-heptane (3) non-polar solvents.

According to the theoretical calculations of the solvent shifts of 4-nitroanisole and betaine-30 dissected into the contributions from inductions (ind), permanent dipole interactions (perm), and dispersion forces (disp) reported by Matyushov et $a l^{10}$ the following observations can be made:

(i) For the solvents that show the major positive differences between theoretical paired parameters the main contribution to the solvation effects can be ascribed to the dipole-dipole solute-solvent interactions. An important role is also played by the dispersion forces on the $E_{\mathrm{T}}{ }^{\mathrm{N}}$ theor values (except for the polar solvent nitroethane (35) for which the permanent dipole solvation is the main contribution: ${ }^{10}\left[\pi^{*}\right.$ theor: perm $>$ ind $\approx$ disp] and $\left[E_{\mathrm{T}}{ }^{\mathrm{N}}\right.$ theor: perm $>$ disp $>$ ind] .

(ii) For the solvents that exhibit the major negative differences between theoretical paired parameters, the dispersion interactions is the main component of the solvation effects of the nonpolar solvents on both, $\pi^{*}$ theor and $E_{\mathrm{T}}{ }^{\mathrm{N}}$ theor values [disp>ind] (with the exception of chlorinated solvents 18 and 19 for which the induction forces are relevant on $\pi^{*}$ theor [ind $>$ disp]). As it is known, the two reference indicators are very different in size and they are expected to be differently sensitive to dispersion forces increasing with solute size. ${ }^{15,16}$

At this point and in order to advance in the comparison of both scales it is now of interest to mention the degree of agreement between the experimental and the theoretical property values quantified through each one of the scales.

\section{Agreement between $E_{\mathrm{T}}{ }^{\mathrm{N}}$ and $E_{\mathrm{T}}{ }^{\mathrm{N}}$ theor}

The results $(\mathrm{N}=55)$ show that (a) the average of the differences is close to zero (bias $=0.069)$; and (b) the limits of agreement are high as a consequence of the fact that the standard deviation is high. These data reveal that, in this comparison, the published/experimental $E_{\mathrm{T}}{ }^{\mathrm{N}}$ values do not agree with the theoretical ones. Moreover, there is no linear correlation $(r=0.562)$ between the experimental and theoretical parameters. As can be seen in Table 1 and Figure 1 , the $E_{\mathrm{T}}{ }^{\mathrm{N}}$ values corresponding to the protic solvents (45-55) are markedly higher than the $E_{\mathrm{T}}{ }^{\mathrm{N}}$ theor ones. As it is well known the $E_{\mathrm{T}}(30)$ parameter includes specific solvent acidity effects whereas the predicted $E_{\mathrm{T}}(30)_{\text {theor }}$ seeks for to minimize the contamination by $\mathrm{H}$-bonding. In particular, the solvents whose positive differences are outside the agreement limits are $\mathrm{H}_{2} \mathrm{O}$ (55), n-hexanol (50), $n$ pentanol (49) and $n$-butanol (48). As can be seen in Matyushov et al. 's data, ${ }^{10}$ the contribution of the nonspecific interactions is dominated by the permanent dipole forces in the case of (55) $\left[E_{\mathrm{T}}{ }^{\mathrm{N}}\right.$ theor: perm $>$ disp $>$ ind $]$, and directed by the dispersion forces in the case of solvents (50), (49), 
and (48) solvents $\left[E_{\mathrm{T}}^{\mathrm{N}}\right.$ theor: disp $>$ perm $>$ ind]. An analysis that includes the nonspecific solute/solvent contributions to $E_{\mathrm{T}}(30)$ has been presented. ${ }^{17}$

Taking into account what is stated above, the B-A approach was applied excluding the protic solvents $(\mathrm{N}=44)$. The results (Table 2, Figure 1) show that the degree of agreement between the $E_{\mathrm{T}}{ }^{\mathrm{N}}$ and $E_{\mathrm{T}}{ }^{\mathrm{N}}$ theor parameters is improved (bias $\left.=-0.044 ; \mathrm{LA}=-0.208 / 0.199\right)$, the linear correlation $(r=0.586)$ being poor. Moreover, when the solvents whose differences are outside or close to the agreement limits [nitroethane (35), 1,2-dichloroethane (22), pyridine (17), iodobenzene (11), BuOAc (31) and propionitrile (33)] are also excluded $(\mathrm{N}=38)$, the $\mathrm{B}-\mathrm{A}$ results reveal that there is agreement between the theoretical and the experimental parameters: the bias is close to zero $(-0.0086)$ and the agreement limits $(-0.117 / 0.099)$ are acceptable. On the other hand, the linear correlation between them is strongly improved $(r=0.924)$.

In connection with this, it is again clearly reflected that there exists real contamination of the $E_{\mathrm{T}}(30)$ parameter with the specific effects. On the other hand, regarding the nonprotic excluded solvents, in the Matyushov's data referred to the participation of the different solvation interactions in the overall solvent effects, it can be observed that:

(a) For the polar nonprotic solvent nitroethane (35) (which exhibits the maximum positive difference) $\left[E_{\mathrm{T}}{ }^{\mathrm{N}}\right.$ theor: perm $>$ ind $>$ disp $]$ the induction component is the major among all the explored solvents, the permanent dipole component being one of the highest [only overcome by nitromethane (34), acetonitrile (32), $\mathrm{PC}(43)$ and $\mathrm{H}_{2} \mathrm{O}$ (55)]. On the other hand, the dispersion component is the lowest of all. With respect to 1,2-DCE (22) and pyridine (17), the contribution of the permanent dipole interactions is also important although the dispersion forces have some influence $\left[E_{\mathrm{T}}{ }^{\mathrm{N}}\right.$ theor: perm $>$ disp $>$ ind $]$.

(b) For the polar non-protic solvent butylacetate (BuOAc) (which exhibits the maximum negative difference), the contribution of the dispersion component is very important, the role of the inductive and permanent dipole components being low $\left[E_{\mathrm{T}}{ }^{\mathrm{N}}\right.$ theor: disp $>$ perm $>$ ind $]$. A similar observation can be made regarding iodobenzene.

In order to continue this comparison study and taking into account that the 4-nitroanisole is only one of the solutes used in the quantification of the KAT dipolarity-polarizability scale, similar analyses were carried out with the published, experimental and theoretical parameters.

\subsection{Agreement between $\pi^{*}$ and $\pi^{*}{ }_{\exp }$}

The results obtained by the application of the B-A method to the data reported in Table 1 (the data corresponding to $\pi_{\text {exp }}$ values for protic solvents have not been reported) indicate that an acceptable degree of agreement exists between the published and the experimental property values (bias $=0.023 ; \mathrm{AL}=-0.069 / 0.115 ; \mathrm{N}=40$ ) although the plot in Figure 1 reveals that the differences for $\mathrm{CHCl}_{3}(19)$ and nitrobenzene (15) are clearly outside the agreement limits. When these two solvents are excluded the convergence is excellent (bias $=0.024 ; \mathrm{AL}=-0.049 / 0.098$; $\mathrm{N}=38$ ). In addition, the linear correlation is very good. 
In this context and taking into account that the published $\pi^{*}$ values are those most frequently used by chemists, the comparative study goes on involving the published KAT scale values. However, caution should be taken in this comparison with the two solvents cited above and also with the protic solvents.

\subsection{Agreement between $\pi^{*}$ and $\pi^{*}$ theor}

The results (Table 2 and the B-A plot included in Figure 1) (N=55) appear unsatisfactory. All the differences are positive and the bias value (0.342) is markedly high revealing that all the published $\pi^{*}$ values are higher than those of $\pi^{*}$ theor. On the other hand, the results show poor linear correlation $(r=0.863)$ between the parameters.

Keeping in mind what was previously said with respect to the published and experimental values and in order to confirm the said behavior, the degree of agreement between $\pi^{*}{ }_{\exp }$ and $\pi^{*}{ }_{\text {theor }}$ was additionally investigated. The results obtained for $\mathrm{N}=44$ [ $\operatorname{bias}(S D)=0.340(0.185)$; $\mathrm{LA}=-0.022 / 0.703 ; r=0.867]$ are similar to those calculated for the comparison of $\pi^{*}$ and $\pi^{*}$ theor. All these results show that the magnitude of the positive differences between $\pi^{*}$ or $\pi^{*}{ }_{\exp }$ and $\pi^{*}$ theor paired measurements is significant. The corresponding plots in Figure 1 clearly reveal that the biggest differences are exhibited by the aromatic [ $\pi^{*}{ }_{\text {theor: }}$ ind $\approx$ disp $>$ perm $]$ and polychlorinated solvents [combinations of the three contributions]. The benzonitrile (16) and nitronenzene (15) solvents are the exceptions to this behavior [ $\pi^{*}$ theor: perm $>>$ disp $>$ ind].

Finally and in view that in all cases the published $\pi^{*}$ values are higher than the theoretical ones, an attempt was made to adjust them by the subtraction of the corresponding bias value ( 0.342 for $\mathrm{N}=55)$. Now, the comparison of the obtained data with $E_{\mathrm{T}}{ }^{\mathrm{N}}$ (including all the solvents) reveals that, although the bias is close to zero (-0.0146), the limits of agreement are high ($0.526 / 0.495)$.

Furthermore, the degree of agreement between the investigated scales was assessed considering each type of solvent set individually (non-polar, aromatic, halogenated, polar aprotic, and protic solvents). The B-A data are presented in Table 3. The results show that there is no agreement between the compared parameters with the exception of $\pi^{*}$ vs $E_{\mathrm{T}}{ }^{\mathrm{N}}$ and $E_{\mathrm{T}}{ }^{\mathrm{N}}$ vs $E_{\mathrm{T}}{ }^{\mathrm{N}}$ theor for non-polar solvents whose values compare closely (it can be pointed out that in both cases the property values are not linearly related). 
Table 3. Bland-Altman results and linear correlation data corresponding to each type of solvents individually

\begin{tabular}{|c|c|c|c|}
\hline \multirow[t]{2}{*}{ Solvents $(\mathrm{N})$} & \multicolumn{2}{|c|}{ Difference vs average } & \multirow{2}{*}{$\begin{array}{c}\text { Linear correlation } \\
r(S D)\end{array}$} \\
\hline & $\operatorname{Bias}(S D)$ & Limits of agreement & \\
\hline \multicolumn{4}{|c|}{$\pi^{*}$ vs $E_{\mathrm{T}}^{\mathrm{N}}$} \\
\hline Nonpolar (7) & $-0.023(0.050)$ & $-0.121 / 0.076$ & - \\
\hline Aromatic (10) & $0.529(0.104)$ & $0.326 / 0.732$ & $0.908(0.081)$ \\
\hline Chlorinated (6) & $0.406(0.184)$ & $0.045 / 0.767$ & $0.702(0.175)$ \\
\hline Polar aprotic $(21)$ & $0.364(0.125)$ & $0.118 / 0.609$ & $0.875(0.106)$ \\
\hline Protic (11) & $-0.091(0.077)$ & $-0.245 / 0.064$ & $0.928(0.078)$ \\
\hline \multicolumn{4}{|c|}{$\pi^{*}$ theor $v s E_{\mathrm{T}}^{\mathrm{N}}$ theor } \\
\hline Nonpolar (7) & $-0.305(0.089)$ & $-0.481 /-0.131$ & - \\
\hline Aromatic (10) & $0.051(0.295)$ & $-0.528 / 0.630$ & $0.671(0.282)$ \\
\hline Chlorinated (6) & $-0.117(0.270)$ & $-0.647 / 0.413$ & - \\
\hline Polar aprotic (21) & $0.089(0.219)$ & $-0.339 / 0.517$ & - \\
\hline Protic (11) & $-0.033(0.135)$ & $-0.299 / 0.232$ & $0.860(0.118)$ \\
\hline \multicolumn{4}{|c|}{$E_{\mathrm{T}}{ }^{\mathrm{N}} v s E_{\mathrm{T}}{ }^{\mathrm{N}}$ theor } \\
\hline Nonpolar (7) & $-0.049(0.040)$ & $-0.129 / 0.029$ & - \\
\hline Aromatic (10) & $-0.004(0.105)$ & $-0.209 / 0.202$ & - \\
\hline Chlorinated (6) & $0.006(0.141)$ & $-0.272 / 0.283$ & - \\
\hline Polar aprotic (21) & $0.007(0.109)$ & $-0.206 / 0.221$ & $0.704(0.084)$ \\
\hline Protic (11) & $0.362(0.080)$ & $0.205 / 0.519$ & $0.874(0.081)$ \\
\hline \multicolumn{4}{|c|}{$\pi^{*} v s \pi^{*}$ theor } \\
\hline Nonpolar (7) & $0.233(0.061)$ & $0.113 / 0.353$ & - \\
\hline Aromatic (10) & $0.474(0.202)$ & $0.077 / 0.871$ & $0.927(0.072)$ \\
\hline Chlorinated (6) & $0.528(0.256)$ & $0.026 / 1.031$ & - \\
\hline Polar aprotic (21) & $0.282(0.102)$ & $0.081 / 0.483$ & $0.938(0.076)$ \\
\hline Protic (11) & $0.305(0.041)$ & $0.226 / 0.385$ & $0.986(0.034)$ \\
\hline
\end{tabular}

\section{Conclusions}

The B-A approach is a useful tool for the comparison of the two selected scales (it focuses exclusively on the differences) constituting an alternative to the correlation analysis. The B-A results allow us to make the following observations: 
- As it was expected, there is no agreement between $\pi^{*}$ and $E_{\mathrm{T}}{ }^{\mathrm{N}}$ paired values. The general tendency shows that $\pi^{*}>E_{\mathrm{T}}{ }^{\mathrm{N}}$ (with the exception of some non-polar solvents and most of the protic ones).

- There is poor agreement between $\pi^{*}$ theor and $E_{\mathrm{T}}{ }^{\mathrm{N}}$ theor. Although the bias is close to zero, the AL reflect a great variation of the differences.

- When all the solvents are included in the analysis, the results show that the degree of agreement between $E_{\mathrm{T}}{ }^{\mathrm{N}}$ and $E_{\mathrm{T}}{ }^{\mathrm{N}}$ theor is not acceptable (the bias is close to zero but the AL are significant). Nevertheless, $E_{\mathrm{T}}{ }^{\mathrm{N}}$ compares closely with $E_{\mathrm{T}}{ }^{\mathrm{N}}$ theor when nitroethane, 1,2dichloroethane, pyridine, butylacetate, iodobenzene and also the protic solvents are excluded.

- The comparison of the published, experimental and theoretical KAT's parameters shows that $\pi^{*}$ or $\pi^{*} \exp$ measurements do not agree with the $\pi^{*}$ theor ones. As can be seen, in all cases the experimentally determined values are higher than the theoretically calculated ones.

- When the B-A comparison approach is applied to solvents of the same type the results show that, even assessing in this way, the general trend is that there is no agreement between the compared measurements. This fact confirms that the solvent effects are consequence of a complex combination of all solute-solvent chemical interactions to molecular level. In connection with this and taking into account that, for the scales under scrutiny, the contributions of those components of the overall solvent effect due to induction, permanent dipoles and dispersion forces were evaluated, ${ }^{9}$ a new perspective could be possible by grouping the explored solvents on the basis of their most relevant individual contribution.

The results presented here are good evidence of the fact that the scales involved in this analysis are dependent on the type of probe and method used to develop each scale. This fact means that, in the first instance, solute-independent parameters could not be experimentally determined in this way, using solvatochromic probe molecules of different molecular structure. Nevertheless, it is a quite a useful analysis tool since it facilitates the extraction of information on the 'medium effects'.

\section{Experimental Section}

General Procedures. The data treatment was carried out using the GraphPad Prism Version 4.0.

\section{Acknowledgements}

This work received financial support from the Universidad Nacional del Litoral, Santa Fe, Argentina (CAI+D Program, Projects: 2005-00745 and 2006-33182/33183). 


\section{References}

1. (a) Reichardt, C. Solvents and Solvent Effects in Organic Chemistry; VCH: Weinheim, 1990. (b) Reichardt, C. Chem. Rev. 1994, 94, 2319. (c) Catalán, J. In: Handbook of Solvents; Wypych, G. Ed.; Chem. Tec. Publishing, 2001; p 583.

2. (a) Dimroth, K.; Reichardt, C.; Siepmann, T.; Bohlmann, F. Liebigs Ann. Chem.1963, 1, 661; (b) Brooker, L. G. S.; Craig, A. C.; Heseltine, D. W.; Jenkins, P. W.; Lincoln L. L. J. Am. Chem. Soc. 1965, 87, 2443. (c) Walter, D. J. Prakt. Chem. 1974, 316, 604. (d) Brownstein, S. Can. J. Chem. 1960, 38, 1590. (e) Kosower, E. M. An Introduction to Physical Organic Chemistry, Wiley: New York, 1968, p 2930. (f) Dubois, J. E.; Bienvenûe, A. J. Chem Phys. 1968, 65, 1259. (g) Allerhand, A.; Schleyer, P. J. Am. Chem. Soc. 1963, 85, 371. (h) Knauer, B. R.; Napier, J. J. J. Am. Chem. Soc. 1976, 98, 4395. (i) Dong, D. C.; Winnik, M. A. Can. J. Chem. 1984, 62, 2560. (j) Gutmann, V. Chemtech 1977, 255 (and refences cited therein). (k) Kamlet, M. J.; Abboud, J.-L.; Taft, R. W. J. Am. Chem. Soc. 1977, 99, 6027. (1) Buncel, E.; Rajagopal, S. J. Org. Chem. 1989, 54, 798. (m) Drago, R. S. J. Chem. Soc., Perkin Trans. 2 1992, 1827.

3. (a) Koppel, I. A.; Palm, V. A. Reakts. Sposobn. Org. Soedin 1971, 8, 291; Engl. Edn. 1974, 11, 137. Advances in Linear Free-Energy Relationships, Ed. Chapman, N. B.; Shorter, J.; Plenum Press: London, 1972, Ch. 5. (b) Krygowsky, T. M.; Fawcett W. R. J. Am. Chem Soc. 1975, 97, 2143. (c) Dougherty R. C. Tetrahedron Lett. 1975, 385. (d) Fowler, F. W.; Katritzky, A. R.; Rutherford, R. D. J. Chem. Soc. B 1971, 460. (e) Swain, C. G; Powell, A. L.; Alunni, S. G. J. Am. Chem. Soc. 1984, 105, 502. (f) Kamlet, M. J.; Abboud, J. L.; Taft, R. W. J. Am. Chem. Soc. 1977, 99, 6027.

4. (a) Abraham, M. H. J. Phys. Org. Chem. 1993, 6, 660. (b) Pure Appl. Chem. 1993, 65, 2503.

5. (a) Catalán, J.; López, V.; Pérez, P.; Martín-Villamil, R.; Rodríguez, J.-G. Liebigs Ann. 1995, 241. (b). Catalán, J.; Díaz, C.; López, V.; Pérez, P.; Paz, J.-L. G.; Rodríguez, J. G. Liebigs Ann. 1996, 1785. (c) Catalán, J.; Díaz, C.; Liebigs Ann. 1997, 1941.

6. (a) Katritzky, A. R.; Karelson, M.; Lobanov, V. S. Pure \& Appl. Chem. 1997, 245. (b) Katritzky, A. R.; Lobanov, V. S.; Karelson, M. Chem. Soc. Rev. 1995, 24, 279.

7. (a) Katritzky, A. R.; Tamm, T.; Wang, Y.; Sild, S.; Karelson, M. J. Chem. Inf. Comput. Sci. 1999, 39, 684. (b) Katritzky, A. R.; Tamm, T.; Wang, Y.; Karelson, M. J. Chem. Inf. Comput. Sci. 1999, 39, 692.

8. (a) Katritzky, A. R.; Fara, D. C,; Hong, Y; Tamm, K.; Tamm, T.; Karelson, M. Chem. Rev. 2004, 104, 175. (b) Katritzky, A. R.; Fara, D. C.; Kuanar, M.; Hur, E.; Karelson, M. J. Phys. Chem. A 2005, 109, 10323.

9. Catalán, J. J. Org. Chem. 1997, 62, 8231.

10. Matyushov, D. V.; Schmid, R.; Landansyl B. M. J. Phys. Chem. B 1997, 101, 1035.

11. (a) Mancini, P. M.; Bock, A; Adam, C.; Pérez, A. del C.; Vottero, L. R. ARKIVOC 2003, (x), 373. (b) Mancini, P. M.; Vottero, L. R. J. Phys. Org. Chem. 2006, 19, 34. 
12. (a) Altman, D. G.; Bland, J. M. The Statistician 1983. 32, 307. (b) Bland, J. M.; Altman, D. G. Lancet 1986, (i), 307.

13. Marcus, Y. Chem. Soc. Rev. 1993, 409.

14. Drago, R. S. J. Org. Chem. 1992, 57, 6547.

15. Abboud, J.-L. M.; Notario, R. Pure Appl. Chem. 1993, 4, 645 and references cited therein.

16. Laurence, C.; Nicolet, P.; Dalati, T.; Abboud, J.-L. M.; Notario, R. J. Phys. Chem. 1994, 98, 5807.

17. Krygowski, T. M.; Wrona, P. K.; Zielkowska, U.; Reichardt, C. Tetrahedron 1985, 41, 4519. 